\section{ENFERMEDADES TIROIDEAS EN PERSONAS CON SÍNDROME DE DOWN EN EL DEPARTAMENTO DE LA PAZ - BOLIVIA}

\author{
Thyroid diseases in people with Down syndrome in the state of La Paz \\ - Bolivia.
}

Carmiña Garcia de Salgueiro ${ }^{1}$ - Beatriz Luna Barron², Norka Hauzateng Sotomayor ${ }^{1}$, Darinka Contreras Castro ${ }^{2}$, Rolndo Paz ${ }^{2}$, Jose Liders Burgos Zuleya ${ }^{3}$, Gonzalo Taboada Lopez², Claudia Heredia Chucantiny ${ }^{1}$

\section{RESUMEN}

El Síndrome de Down está asociado a varias formas de distiroidismo, el hipotiroidismo es la más común en esta población. La alteración en la función tiroidea puede aumentar la morbilidad de este síndrome, disminuyendo la calidad y expectativa de vida en estos pacientes.

El objetivo del presente estudio fue describir el estado de la función tiroidea en pacientes con Síndrome de Down de la fundación Aywiña y la Fundación Down Bolivia, del departamento de La Paz, mediante la determinación de pruebas bioquímicas del eje tiroideo. El grupo de estudio estuvo comprendido por 30 individuos, 19 del sexo masculino y II del sexo femenino.

Los resultados muestran un $50 \%$ de distiroidismo y la población con mayor incidencia de alteración tiroidea fueron los menores a 13 años. La principal patología fue el hipotiroidismo subclínico (24\%), seguido del hipotiroidismo subclínico autoinmune. Cabe resaltar que se observó un porcentaje elevado de hipertiroidismo (I3\%), que es superior a lo reportado por otros autores. No se observaron diferencias significativas entre sexos.

La elevada incidencia de distiroidismo hallada, sugiere que la población con Síndrome de Down del departamento de La Paz, es vulnerable a este tipo de trastornos, confirmando lo reportado en otras poblaciones.

Debido a que la presencia de enfermedades tiroidea aumenta las complicaciones de este síndrome, es importante realizar controles anuales que evalúen la función tiroidea y se plantea la necesidad de contar con rangos de referencia para las pruebas bioquímicas del eje tiroideo en la población con Síndrome de Down de nuestro país.

\section{ABSTRACT}

Down Syndrome is associated with several forms of dysthyroidism, hypothyroidism is the most common on this population.Alteration in thyroid function may increase the morbidity of this syndrome, decreasing the quality and life expectancy in these patients.

The main objective of the present study was describe the state of thyroid function in patients with Down Syndrome of the Aywiña Foundation and the Down Bolivia Foundation in the department of La Paz, through the determination of biochemical tests of the thyroid axis. The target group were consisted of 30 individuals, for which 19 males and II females.

The obtained results indicate a $50 \%$ of dysthyroidism, the population with the highest incidence of thyroid alteration were those are younger than 13 years. The main pathology was subclinical hypothyroidism (24\%), followed by subclinical autoimmune hypothyroidism. It is noteworthy that a high percentage of hyperthyroidism (13\%) was observed, which is higher than reported by other authors. Also there were no significant differences between genders.

The high incidence of dysthyroidism found suggests that the population with Down Syndrome in the department of La Paz is vulnerable to this type of disorder, confirming what has been reported in other populations.

Due to the presence of thyroid dysfunction increases the complications of this syndrome, it is important to perform annual tests that evaluate thyroid function and the need to have reference ranges for the biochemical tests of the thyroid axis in the population with Down Syndrome of our country.
${ }^{1}$ Instituto SELADIS- Faultad de Ciencias Farmaceuticas y Bioquimicas- UMSA, La Paz -Bolivia.

${ }^{2}$ Instituto de Genetica- Facultad de medicina - UMSA La Paz -Bolivia.

${ }^{3}$ Caja de Salud de la Banca Privada - La Paz -Bolivia.

Correspondencia a: Correo electrónico: clau.h.chucantiny@gmail.com

Palabras clave: Sindrome de Down, Enfermedades de la TIroides, Hipotiroidismo.

Keywords: Down Syndrome, Thyroid Diseases., Hypothyroidism.

\footnotetext{
Procedencia y arbitraje: no comisionado, sometido a arbitraje externo.

Recibido para publicación: 15 de Abril del 2017 Aceptado para publicación: 20 de Junio del 2017

Citar como: Rev Cient Cienc Med 2017;20(1): 11 - 15
} 
E Síndrome de Down, conocido como trisomía del par 21, es una condición genética determinada por una alteración del número de cromosomas, en donde existen 47 cromosomas en lugar de 46 . Se pueden presentar en tres formas: la trisomía 21 libre en un 95\% de los casos, la translocación en un $4 \%$ y el mosaicismo en el $1 \% 1$.

La prevalencia de alteraciones endocrinológicas, generalmente de origen autoinmune, es muy elevada en las personas con síndrome de Down, entre las endocrinopatías asociadas al Síndrome de Down (SD) más frecuentes, están los desórdenes de la glándula tiroidea, los cuáles generalmente tienen un origen autoinmune2. Algunos autores refieren que la suceptibilidad de las personas con SD a desarrollar hipotiroidismo se debe al estrés oxidativo que afecta a la biosíntesis de tiroxina3; pues a nivel molecular se ha observado un incremento de este proceso metabólico relacionado con mayor producción de radicales libres 4 donde el gen SOD1 codificado en el cromosoma 21 estaría sobre expresado resultando en un incremento de especies reactivas de oxigeno (ROS) que llevan a daño oxidativo del DNA jugando un rol importante en la patogénesis de la comorbilidades del SD, tales como disgenesis tiroidea y alteraciones fisiopatológicas de la glándula tiroidea.

Como se mencionó en el párrafo anterior, la disfunción tiroidea más frecuente en las personas con SD es el hipotiroidismo, diferentes estudios muestran porcentajes que oscilan entre el 3\% $y$ el $54 \% 5,6,7$, incluyendo las manifestaciones de hipotiroidismo subclínico y autoinmune, este último caracterizado por una elevación marcada de TSH (Tirotropina) y presencia de anticuerpos antitiroperoxidasa (AcTPO) y/o antitiroglobulina (Ac-Tg). El solapamiento de manifestaciones clínicas en estos pacientes complica su detección a no ser que se realicen pruebas de laboratorio para coadyuvar a su diagnóstico.

Debido a que las hormonas tiroideas tienen numerosas funciones vitales, entre ellas el desarrollo cerebral, es que las complicaciones por la falta o disminución de estas pueden conducir a un daño neurológico irreparable en el paciente, de acuerdo a la duración y gravedad de la deficiencia, aumentando el grado de discapacidad intelectual, retraso en el crecimiento y complicaciones cardiocirculatorias 8,9 .
La detección temprana de alteraciones tiroideas en personas con SD no solo permite prevenir las complicaciones futuras (obesidad, eventos cardiovasculares) sino también mejorar su calidad de vida, ya que es posible minimizar los efectos a nivel de desarrollo intelectual en estos pacientes si se detectan en etapas tempranas de la niñez.

Bolivia es una de las zonas endémicas en deficiencia de yodo9,10, y en consecuencia el hipotiroidismo es una de las principales causas de deficiencia intelectual (incidencia de 1:1170 recién nacidos vivos)9, a pesar de esto, no se han realizado estudios epidemiológicos sobre la función tiroidea en personas con SD en el país, aun cuando se ha comprobado, en diferentes estudios que esta es una población vulnerable5, 11 .

Debido a la alta incidencia de afecciones de la glándula tiroides en nuestro medio y la poca información que existe al respecto en la población con Síndrome de Down, este trabajo tiene como objetivo describir el estado de la función tiroidea en pacientes con SD de la fundación Aywiña y la Fundación Down Bolivia del departamento de La Paz, mediante pruebas bioquímicas de laboratorio.

\section{MATERIALES Y MÉTODOS}

Tipo de estudio: descriptivo, transversal.

Universo: no se cuenta con un registro oficial del número de personas con SD en el departamento de La Paz, sin embargo se estima que son 2500 las personas que lo padecen.

Tamaño de mMuestra: En este trabajo la población total fue de 33 pacientes con Síndrome de Down, pertenecientes a la fundación Aywiña y Fundación Down Bolivia del departamento de La Paz. La edad de los pacientes estuvo comprendida entre los 1 a 35 años. Tres de los pacientes fueron descartados debido a que las muestras de sangre no cumplieron los criterios de aceptación para su procesamiento. Al resto de los participantes del estudio se evaluó el perfil tiroideo completo: TSH, T3, T4, T4 libre y la determinación de anticuerpo anti-TPO y anti$\mathrm{Tg}$

Criterios de Inclusión: Personas diagnosticadas con SD que asistieron a la Fundacion Aywiña y la Fundacion Down Bolivia durante los me 
ses Abril a Septiembre de 2015 y cuyos padres, madres o tutores hayan aceptado su participación dentro del estudio.

Criterios de Exclusión: Personas diagnosticadas con SD cuyos padres, madres o tutores no aceptaron su participación dentro del estudio.

Las determinaciones hormonales se realizaron tomando una muestra de sangre venosa por venopunción, después de la centrifugación de la muestra, el suero fue almacenado a $-20^{\circ} \mathrm{C}$ hasta su procesamiento.

Se determinó la concentración del perfil tiroideo completo: TSH, T3, T4 y T4 libre, por el método de quimioluminiscencia en el equipo IMMULITE $^{\circledR} 1000$ de la marca SIEMENS, y los Anticuerpos anti Tiroperoxidasa (Ac-TPO) y anti Tiroglobulina (AcTg), por la técnica de ELISA, utilizando el kit comercial de la marca MonoBind. Los resultados obtenidos se analizaron utilizando el programa SPSS ${ }^{\circledR}$ v 18.0.

Para el estudio ecográfico, se realizó con el paciente en decúbito dorsal pasivo, se procedió al estudio con sonda convexa multifrecuencia en la región cervical anterior para identificar la glándula tiroides para su medición, aplicación de doppler color y búsqueda de lesiones.

RESULTADOS

La población estudiada estuvo compuesta por 33 personas con Síndrome de Down, que asistieron a la fundación Aywiña y la fundación Down Bolivia del departamento de La Paz, durante los meses de abril a septiembre del año 2015. Del total de la población el 19 (63\%) eran del sexo masculino y 11 (37\%) del sexo femenino.

El 55\% de los pacientes estuvo en el rango de edad de 1 - 12 años, 15\% entre 13 -18 años, el $27 \%$ entre 19 -30 años y el 3\% mayor a 30 años; con una media de 11 años para varones y 21,3 en mujeres.

Se encontró que el 50\% de la población estudiada tiene alteración tiroidea en cualquiera de sus patologías específicas y el 50\% presentó perfiles tiroideos normales (dentro de los rangos de referencia).

Se clasificaron las alteraciones tiroideas según los siguientes patrones, obtenidos del compendio de rangos de referencia del equipo IMMULITE $^{\circledR}$ e IMMULITE ${ }^{\circledR} 2000$ - Siemens; en relación a las concentraciones de los analitos estudiados y se obtuvieron obtuvo los resultados observados en la figura 1 se clasificó las alteraciones tiroideas (figura 1).

Patrón 1 "Eutiroideo": TSH, T3, T4, T4 Libre dentro del rango normal, sin presencia de anticuerpos anti-TPO y anti-TG

Patrón 2 "Hipotiroidismo primario": TSH elevada; T3, T4 y T4 libre por debajo del rango normal, sin presencia de anticuerpos anti-TPO y anti-TG.

Patrón 3 "Hipotiroidismo subclínico": TSH elevada; T3, T4 y T4 libre dentro del rango normal, sin presencia de anticuerpos anti-TPO y anti-TG.

Patrón 4 "Hipotiroidismo subclínico autoinmune": TSH elevada; T3, T4 y T4 libre dentro del rango normal, con títulos elevados de anticuerpos anti-TPO y anti-TG.

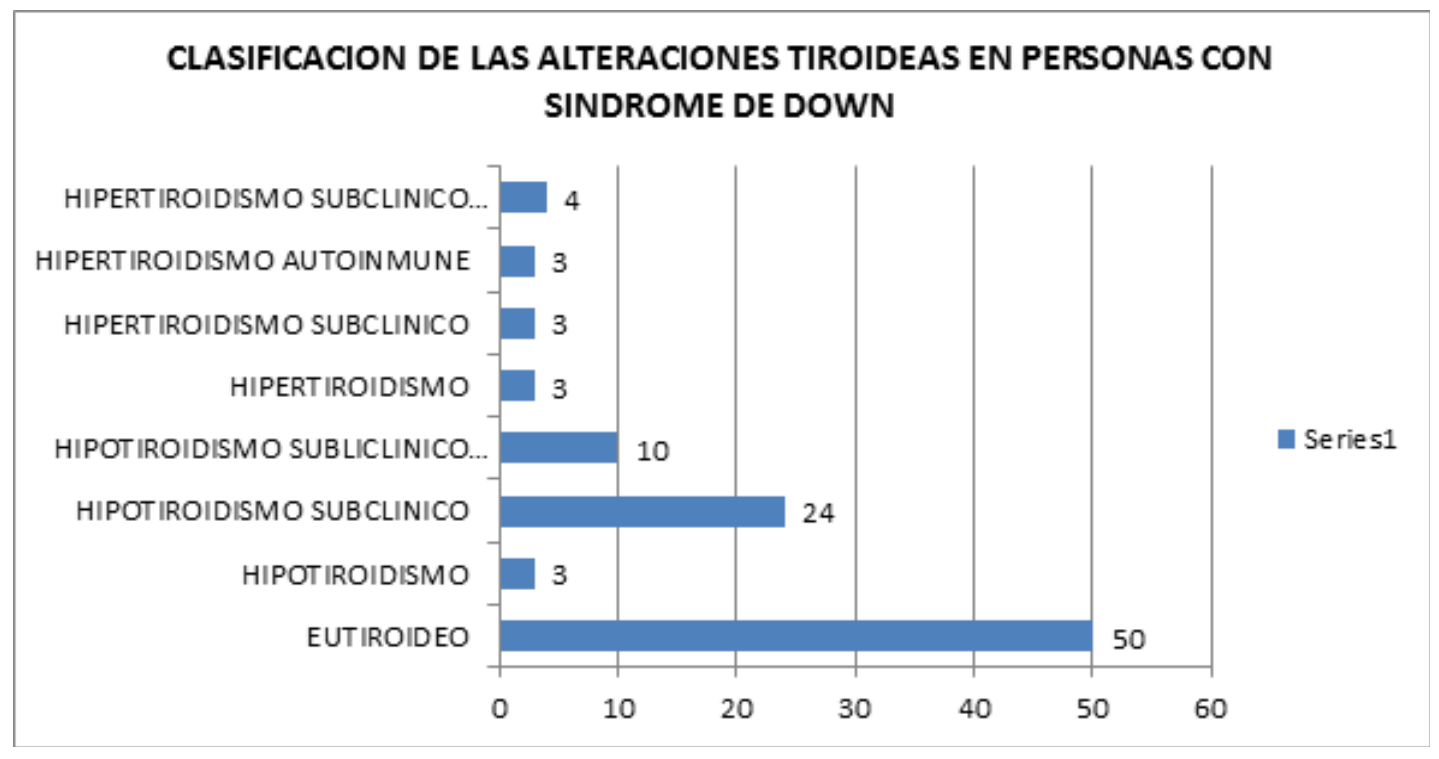


Patrón 5 "Hipertiroidismo": TSH por debajo del rango normal; T3, T4 y T4 libre por superiores al rango normal, sin presencia de anticuerpos anti-TPO y anti-TG.

Patrón 6"Hipertiroidismo subclínico": TSH por debajo del rango normal; T3, T4 y T4 libre dentro del rango normal, sin presencia de anticuerpos anti-TPO y anti-TG.

Patrón 7 "Hipertiroidismo subclínico autoinmune": TSH disminuida; T3, T4 y T4 libre dentro del rango normal, con títulos elevados de anticuerpos anti-TPO y anti-TG.

Patrón 8 "Hipertiroidismo autoinmune": TSH disminuida; T3, T4 y T4 libre elevadas, con títulos elevados de anticuerpos anti-TPO y anti-TG. 6

De la población identificada con alteración tiroidea, un $47 \%(n=7)$ ya tenía diagnóstico clínico previo de distiroidismo, que fue confirmado en el presente estudio. El restante $53 \%(n=8)$ fue diagnosticado con algún tipo de distiroidismo mediante las pruebas de laboratorio y ecografía realizadas en este estudio.

Si excluimos del estudio a aquellos pacientes identificados previamente con alguna alteración tiroidea y que siguen un tratamiento de la población total con distiroidismo, en este trabajo se encontró que un 35\% de la población total $(n=30)$ presenta alteraciones tiroideas.

Según sexo, el $44 \%$ de los pacientes varones y $55 \%$ de las pacientes mujeres presentan alteración tiroideas; en ambos casos la principal patología tiroidea es el hipotiroidismo subclínico (mujeres $=18 \%$ y hombres $=26 \%$ ).

En los resultados del estudio ecográfico se observó que el volumen total de la glándula tiroides esta disminuida de manera general especialmente en pacientes mayores a los 20 años con volúmenes de 3,1 - 3,5 cc (Volumen normal total 6-9 cc. 3) (Tabla Nº1).

\section{DISCUSIÓN}

Sarici y colaboradores (2012), realizaron un estudio en neonatos con Síndrome de Down, hallando que el 53,8\% de la población estudiada $(n=50)$ tenían disfunción tiroidea. Debida principalmente a hipertirotropinemia (36 pacientes) y deficiencia de iodo (12 pacientes). El presente estudio, presento que del total de individuos estudiados $50 \%$ presento alteraciones tiroideas de los cuales el 15\% tenía un diagnóstico previo de alteración tiroidea y sigue un tratamiento para su enfermedad, el 35\% de pacientes fueron diagnosticados con patología tiroidea a través de las pruebas realizadas en este trabajo.

Por otro lado, nuestros resultados reflejan que el hipotiroidismo subclínico fue la principal alteración tiroidea observada en este grupo (24\%), lo cual concuerda con trabajos previamente reportados, tal como el que refiere Jimenez-Lopez y colaboradores (2001) que hallaron en una población de 48 niños un 45,8\% de casos de hipotiroidismo subclínico y Shaw (2006) que realizo un estudio prospectivo donde encontró un $15,6 \%$ de hipotiroidismo en la población, donde el $12,5 \%$ se compensó durante el tiempo de estudio.

Un dato que llama la atención en este estudio es el alto porcentaje de hipertiroidismo (13\%), en comparación de otros trabajos que reportan frecuencias de un 1,7\%6 y casos aislados de hipertiroidismo12 lo que sugiere que nuestra población podría tener mayor susceptibilidad a sufrir esta afección tiroidea, sin embargo, es necesario realizar el seguimiento de los pacientes que nos permita identificar los posibles factores causantes de esta alta frecuencia de hipertiroidismo.

\begin{tabular}{|c|c|c|c|c|c|c|}
\hline Edad & $\mathrm{M}$ & $\mathrm{F}$ & $\mathrm{N}^{\circ}$ de casos & Rango de Vol. total & Media & SD \\
\hline $1-10$ años & 14 & 2 & 19 & $1,1-2,6$ & 1,85 & 0,4 \\
\hline $11-20$ años & 6 & 1 & 8 & $3,3-4,3$ & 3,8 & 0,7 \\
\hline 21-30 años & 1 & 2 & 4 & $3,1-3,5$ & 3,3 & 0,6 \\
\hline Mayor 30 años & 2 & 0 & 2 & $2,9-3,2$ & 3,05 & 0,4 \\
\hline
\end{tabular}

según sexo, no se hallaron diferencias significativas entre hombres y mujeres (mujeres $=18 \%$ y hombres $=26 \%$ ), resultados similares a los reportados por Jimenez-Lopes el 2001.
Los resultados obtenidos muestran similitud a lo reportado por otros autores en diferentes regiones5,11, resaltando una alta predisposición a los desórdenes tiroideos en niños con SD 
menores de 12 años.

En el estudio ecográfico encaminado a evaluar dimensiones de la glándula tiroidea y/o alteraciones estructurales se observó un volumen disminuido en la mayoría de los casos estudiados, independientemente de la edad y el sexo, de acuerdo con Cebecy y col.13 se plantea una probable relación de alteraciones tiroideas y disgenesias de la glándula como eventos en cascada. Esta observación estaría relacionada a alteraciones de producción y estimulación hormonal.

García-España (2014), evaluó el perfil tiroideo en personas sanas con SD, teniendo como parámetro bioquímico el valor de la TSH para definir la función tiroidea, mostrando que los valores de TSH se encuentra dos desviaciones estándar por encima de la población general. Concluyendo la necesidad de definir criterios de normalidad e hipotiroidismo subclínico en la población con SD y así proponer nuevas pautas para el diagnóstico y tratamiento6. En nuestro país, no se realizaron estudios, en cuanto a la función tiroidea en personas con SD, pero se cuentan con escasos datos de la incidencia de hipotiroidismo congénito en población en general9, que muestran que la incidencia de hipotiroidismo congénito es de 1:1170 (RN) cifra elevada con relación a la incidencia mundial (1:2500 a 1:5500).

Si a esta elevada incidencia hipotiroidismo en nuestro país sumamos la mayor predisposición a desarrollar alteraciones tiroideas en los pacientes con SD, se hace necesario y de gran interés para el sistema de salud conocer la frecuencia de distiroidismo y tener valores de normalidad exclusivos para las personas con Síndrome de Down propios de nuestro medio que permitan un diagnostico y tratamiento más certeros, para mejorar la calidad de vida de esta población.

\section{REFERENCIAS}

1. Moreno-Vivot, E. El recién nacido con síndrome de Down. Revista Española de pediatría. Clínica e Investigación. 2012; 68 (6): 404-408. Disponible en: http://www.sindromedown.net/wp-content/uploads/2014/09/122L_revista.pdf 2. Alpera, R., Morata,.J, López, M.J. Alteraciones endocrinológicas en el síndrome de Down. Revista Española de pediatría. Clínica e Investigación. 2012; 68 (6): 440-444. Disponible en: http://www.sindromedown. net/wp-content/uploads/2014/09/122L_revista.pdf 3. Mehar Sulthana, S., Nandha Kumar, S., Sridhar, MG., Vishnu Bhat, B., Ramachandra Rao, K. Oxidative stress in children with Down syndrome. Current Pediatric Research. 2012; 16 (2): 111-114. Disponible en: http://www.alliedacademies.org/articles/ oxidative-stress-in-children-with-down-syndrome.pdf 4. Campos C, Casado Á. Oxidative stress, thyroid dysfunction \& Down syndrome. Indian J Med Res 2015;142:113-119. 5. Sarici, D., Akin, M. A., Kurtoglu, S., Gunes, T., Ozturk, M. A., \& Akcakus, M. Thyroid functions of neonates with Down syndrome. Italian journal of pediatrics. 2012, 38(1), 44. Disponible en: https://ijponline. biomedcentral.com/articles/10.1186/1824-7288-38-44. $6 . \quad$ García-España, F., Gómez-Villegas, I., Perán, M., Sánchez-Jiménez, F., Perán, S. ¿Son aplicables los criterios analíticos generales para definir el hipotiroidismo en personas con síndrome de Down?. Acta Pediatr Esp. 2014; 72(8): 146-151. Disponible en: http://www.actapediatrica.com/ index.php/secciones/originales/1015-son-aplicables-loscriterios-analiticos-generales-para-definir-el-hipotiroidismo-en-personas-con-sindrome-de-down\#.WUsvKvIEnIU 7. Unachak, K., Tanpaiboon, P., Pongprot, Y., Sittivangkul, R., Silvilairat, S., Dejkhamron, P., \& Sudasna, J. Thyroid functions in children with Down's syndrome. Medical journal of the Medical Association of Thailand, 91 (1): 56-61. Disponible en: http://www. thaiscience.info/journals/Article/JMAT/10402407.pdf 8. Claret-Torrents, C., Goday-Arno, A., CerdàEsteve, M., Flores-Le Roux, J., Chillarón-Jordan, J. J., \&
Cano-Pérez, J. F. Hipertiroidismo en el síndrome de Down. Revista Médica Internacional sobre el Síndrome de Down. 2009, 13(1), 2-8. Disponible en: http://www.sciencedirect.com/science/article/pii/S1 138207409700028 9. Queiroz, M. Incidencia de hipotiroidismo congénito, detectado por el método de tamizaje neonatal en el Distrito III de la ciudad de La Paz. ev. Soc. Bol. Ped. 2000; 39 (2): 50-54. Disponible en: https://www2.bago. com.bo/sbp/revista_ped/html/hipotiroidismo_cong.html 10. Mazzi, E., Borth, V. Cribado neonatal. Revista de la Sociedad Boliviana de Pediatria. 2010. 49 (3): 145-149. Disponible en: https://www2.bago.com. bo/sbp/revista_ped/html/hipotiroidismo_cong.html 11. Shaw, C. Thyroid dysfunction in Down syndrome. Kathmandu University Medical Journal. 2006, 4 (2): 182 - 186. Disponible en: http:// imsear.li.mahidol.ac.th/handle/123456789/46525 12. Soriano, L. M. Enfermedad de Graves en pacientes con síndrome de Down. Anales de pediatria. 2003, 58(1): 63-66. Disponible en: http://www.sciencedirect.com/science/article/pii/S1695403303779946 13. Cebeci, A. N., Güven, A., \& Yıldız, M.. Profile of hypothyroidism in Down's syndrome. Journal of clinical research in pediatric endocrinology. 2013, 5(2), 116-120. Disponible en: https://www.ncbi.nlm.nih. gov/pmc/articles/PMC3701917/pdf/JCRPE-5-116.pdf 